\title{
POWER SUPPLY SYSTEM FOR SUPERCONDUCTING MAGNETS AT KEKB-IR
}

\author{
T.Ozaki ${ }^{\#}$, A.Akiyama, Ta.Kubo, T.Nakamura, T.Ogitsu, N.Ohuchi, K.Tsuchiya, M.Yoshida, \\ KEK, Tsukuba, Japan.
}

\section{Abstract}

At KEKB, a pair of superconducting magnets is installed symmetrically with respect to the Tsukuba Interaction Region (IR) in order to focus and steer the beams. Two quadrupole magnets (QCS) are being prepared to focus the beams, two solenoid coils are to shield the magnetic field of the solenoid coil of the detector, and 6 correction coils are to steer the beams. These magnets are to be energized by high-stability power supplies (P.S.s) to maintain a long-term magnetic field. This paper discusses the details concerning the P.S.s and their control.

\section{MAIN P.S. FOR QCS MAGNETS}

In the TRISTAN main ring, 4 pairs of superconducting quadrupoles (QCS) were used at each 4 colliding sections. The 4 P.S. were fabricated [1]. At KEKB, one of them was improved to excite new QCS magnets. The KEKB QCS magnets are compact compared to the TRISTAN QCS magnets. The 2 QCS magnets are powered in series.

The QCS P.S. consists of a main P.S. and an auxiliary P.S. as shown in Fig.1. The main P.S. has a capability of $3500 \mathrm{~A}$ and $15 \mathrm{~V}$. This P.S. consists of a converter and a quench-protection circuit. The converter consists of 12-pulse phase-controlled rectifies, a passive filter and an active filter. A thyristor switch has prepared to intercept the dc current in the case of any quench trouble.

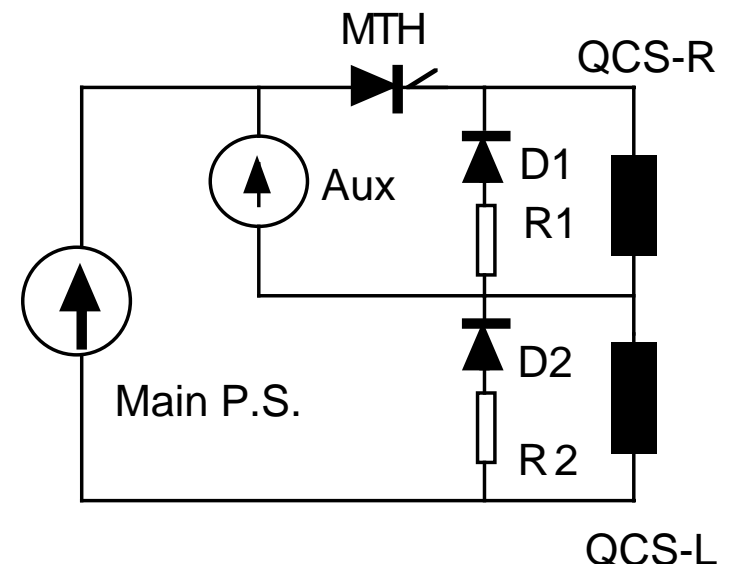

Figure 1: The circuit of QCS main and auxiliary P.S..

\subsection{Effect for New Load}

The inductance of the load becomes $36.4 \mathrm{mH}$ instead of $120 \mathrm{mH}$. The resistance of the cable remains at $1.5 \mathrm{~m} \Omega$.
In the KEKB operation, the QCS magnets is excited to generate the field gradient of $21.3 \mathrm{~T} / \mathrm{m}$ at a current of 2830A. We can therefore use the TRISTAN QCS P.S. with a few improvements.

The transfer-function block diagram of the closed-loop control in the P.S. is given in Fig. 2. The system has two closed control loops. The minor auto-voltage regulation (AVR) loop is inside the auto-current regulation (ACR) loop. The simulations show that the responses become slow. However, it is not serious because KEKB is a storage ring without acceleration.

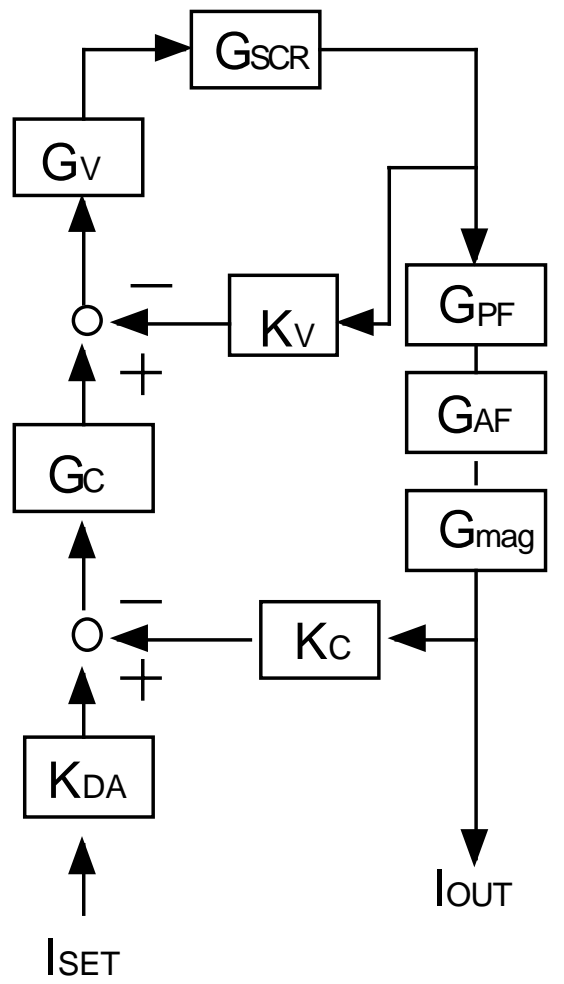

Figure 2: The transfer function block diagram of QCS main power supply is shown. The elements are given as follows:

$$
\begin{aligned}
& G_{S C R}=20 \cdot 1.35 \cdot \frac{1}{8.25} \\
& G_{P F}=\frac{63000}{s^{2}+980 s+63000}, G_{A F}=\frac{1}{0.2 s+1}, \\
& G_{m a g}=\frac{1}{8 s+1}, G_{V}=\frac{1000}{s}, G_{C}=\frac{54.5}{s}+94, \\
& K_{C}=\frac{10}{4000}, K_{D A}=\frac{9.5}{3500} \cdot \frac{35}{40} \cdot \frac{10}{9.5}
\end{aligned}
$$

\# Email: ozaki@ post.kek.jp 


\subsection{Fast-Stop and Slow-Stop}

The fast stop of the QCS P.S. is triggered from one of 3 quench detectors. The thyristor switch (MTH) intercepts the dc current within $1 \mathrm{~ms}$, and the energy stored in the magnets dissipates in stainless-steel resistors ( $R_{1}$ and $\left.R_{2}\right)$ through the diodes as shown Fig.1.

In KEKB, the inductance $\left(L_{1}\right)$ of QCS of the right side is $16.2 \mathrm{mH}$, and $L_{2}$ of the left side is $20.2 \mathrm{mH}$. In order to reduce the current in the neutral line, we changed resistor $R_{1}$ based on the condition $L_{1} / R_{1}=L_{2} / R_{2}$. The resistors are $0.1 \Omega$ and $0.125 \Omega$, respectively.

In the slow-stop mode, a counter IC (which connected to the DAC) counts down by 1 bit per clock pulse. The current decreases at a rate of about $10 \mathrm{~A} / \mathrm{s}$.

\section{AUXILIARY P.S. FOR QCS-R}

A bipolar 40 A auxiliary P.S. is connected to the QCS-R magnet. Such a connection produces an unbalance between two QCS magnets to zero with the required accuracy. On the contrary, it has the possibility to produce an imbalance of the field integrals between two magnets.

The circuit of the auxiliary P.S. is shown in Fig.1. The output terminals of the auxiliary P.S. are connected to a magnet through contactors. The relay contactors are used to change the polarity on the magnet. In minus polarity, the plus voltage of the main P.S. imposes the output of the auxiliary P.S.. To avoid this situation, a series of diodes is installed in line, which produces an offset voltage of $7 \mathrm{~V}$.

\section{P.S. FOR SOLENOID COIL}

The detector BELLE at IR has a solenoid field with 1.5T to distinguish the particle track. The solenoid field couples the horizontal and vertical betatron oscillations of the beam. This makes a ring less dynamically stable and lowers the peak luminosity. The simplest compensation is to place two half-solenoids at each side of the IR. The solenoids produce an axial magnetic $\left(B_{Z}\right)$ field opposite to the detector solenoid.

Superconducting solenoid coils are installed before the first quadrupole QCS. The inductance of the right-side solenoid is $1.44 \mathrm{H}$ and that of the left side is $0.9 \mathrm{H}$. Since the resistances of the cabling from the P.S. to the magnet are $8.3 \mathrm{~m} \Omega$ and $11 \mathrm{~m} \Omega$, the time constants are $173 \mathrm{~s}$ and $82 \mathrm{~s}$, respectively.

\subsection{Switching Power Supply}

In KEKB, many switching P.S.s are installed. We adopted the switching type for the superconducting coils. The ratings of the solenoid P.S. are $650 \mathrm{~A}$ and $30 \mathrm{~V}$. In the P.S., the three-phase input (AC 420V) is rectified and filtered to provide an unregulated dc voltage (DC 600V). The unregulated dc is converted into high-frequency square waves by the IGBTs. The square waves through the highfrequency transformers are rectified. The output voltage of the converter is controlled by the PWM (pulse width modulator) circuit. Power FETs so as to control the output current precisely is used. The current regulator obtains its feedback signal from a high-precision DCCT (DANFYSIK 864I DCCT). This type DCCT is compact and cheap compared to the 4-core-type DCCT used in TRISTAN [2]. The output current must be compared with a current reference. The reference source is a 16-bit DAC on its $9.5 \mathrm{~V} \mathrm{dc}$ scale. The error signal is sent to the FET bank.

\subsection{Fast-Stop}

DCCBs (Direct Current Circuit Breaker) are adopted to intercepts the dc current. The response speed is about 30 ms, which is much slower than the thyristor switch because of opening the mechanical contactors. The operation is limited to 500 times. However, the cost is very cheap compared to that of the thyristor switch. A damping resistor of $0.71 \Omega$ is connected to the terminals through a diode.

At the initial cool-down testing of solenoid coils, the current was limited by quenching at about 210 A. Fig.3 shows the output voltage and current of the ESR P.S.. The current ramps from $200 \mathrm{~A}$ at the speed of $1 \mathrm{~A} / \mathrm{s}$. Around $210 \mathrm{~A}$, the voltage suddenly increases. DCCB intercepts the dc current, the minus voltage of $150 \mathrm{~V}$ is produced, and the current decays at the time constant of 2 sec.. Afterward, the quench origin was revealed, and expeditious remedial measures were taken. At the second cool-down test, no quenching occurred for currents up to $110 \%$ of the design values.

Interference effects between these solenoids and the detector solenoid were measured by the pseudo-quench tests. The peak voltages on the output terminals of the each P.S. when the quench of the detector solenoid occurred were $7 \mathrm{~V}$ and $4.5 \mathrm{~V}$, respectively.

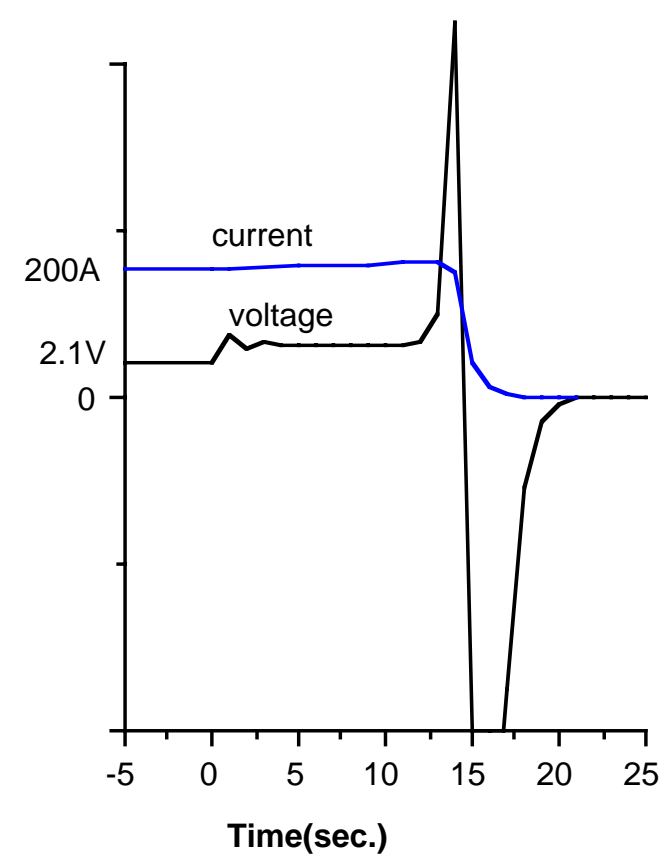

Figure 3: The fast-stop operation of solenoid ESR power supply is shown . 


\subsection{Slow-Stop}

In the slow stop, the gate pulses to fire IGBTs are blocked and the source voltage of the converter is vanished. The current through the magnet naturally decays. The current decreases of the ESR P.S. and ESL P.S. are shown in Fig.4, respectively. The decreases are faster than the exponential decays of the time constant of the load by the nonlinear resistances such as FETs and diodes in the P.S..

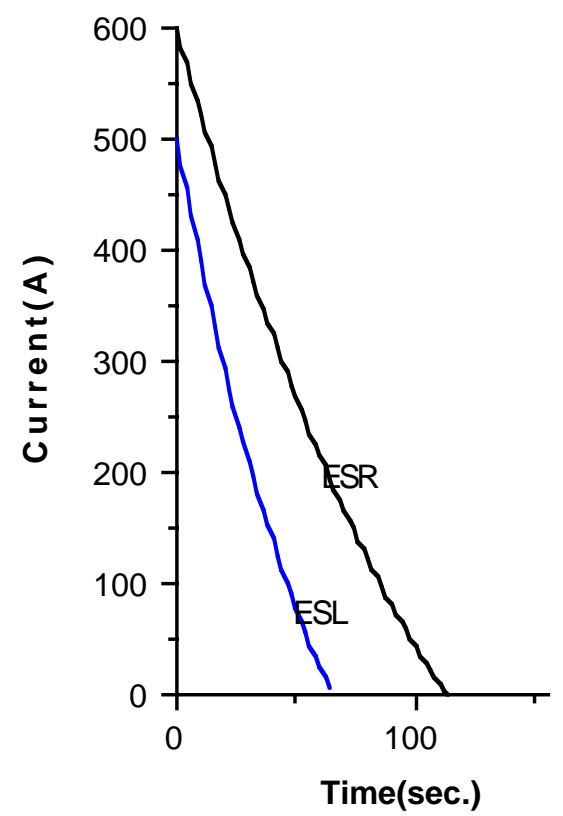

Figure 4: The slow-stop operations of solenoid power supplies are shown.

\section{P.S. FOR CORRECTION COIL}

Correction coils (a skew quadrupole, an H-dipole and a V-dipole) are housed in each of the QCS cryostats. These 6 coils are excited by 6 bipolar P.S.s. The inductance of the skew is $23 \mathrm{mH}$, and that of the steering is $45 \mathrm{mH}$. The rating of the P.S. is $\pm 50 \mathrm{~A}$ and $\pm 20 \mathrm{~V}$. The field strength of the steering coil is about $0.05 \mathrm{~T}$ and the gradient of the skew quadrupole is about $0.4 \mathrm{~T} / \mathrm{m}$.

The terminals of the DC output of a P.S. are connected to the magnet though relay contactors. The relay contactors are used to change the polarity. An energydamping circuit and DCCB is installed between the relay contactors and the magnet.

The current is monitored using two DCCTs. One is for current stabilization control and the other for the monitor of the damping current.

\section{CONTROL SYSTEM}

These P.S.s are operated by computers via VMEARCNET. They are also operating under a safety system. Each P.S. has a fast-stop mode and a slow-stop mode.

\subsection{Current Setting}

The exciting current is remotely set by computers through the VME-ARCNET. The VME-ARCNET is adopted in stead of the CAMAC in TRISTAN. The interface board in each P.S. receives current setting signals from an Input/Output Controller (IOC) in a VME crate and send fault-signals to the IOC. The communication between IOC and interface board employs ARCNET. The VME is connected to the KEKB computer system. The system has utilized EPICS (Experimental Physics and Industrial Control System).

The interface board sends 16-bit digital signals to a DAC (Digital to Analog converter) in the P.S.. The output of the DAC is used as a current reference.

\subsection{Fast-Stop Interlock}

When a quench in a magnet occurs, the exciting current must be quickly decayed. The quench detection is based on measurements of the voltage among the ends and center tap of the coil. If a quench occurs, a differential voltage appears, and the quench is detected. As soon as the detector sends a trigger signal to the P.S., it must be quickly stopped. The typical quench threshold is set at $1 \mathrm{~V}$ for $10 \mathrm{~ms}$ or more.

\subsection{Slow-Stop Interlock}

In some cases, the current must be decreased slowly based on the judgment of the cryogenic computer or the operators. The EX1000 computer which controls the cryogenic system can command the P.S.s to stop either quickly or slowly. In a different way, the P.S.s can be stopped slowly by pressing stop-buttons for rotary warning lights .

\section{CONCLUSIONS}

The power supply system for superconducting magnets at KEKB were designed and fabricated [3]. Since the KEKB commissioning of December of 1998, the superconducting magnet power supply system is under operation.

\section{REFERENCES}

[1] T. OZAKI, K.EGAWA, K. ENDO, N. OHUCHI, Y. OHSAWA, A. KABE, Ta. KUBO, S. KURODA, R. SUGAWARA, K. TSUCHIYA, H. FUKUMA, Y. MORITA and S. OGAWA, " Power Supply System for Superconducting Quadrupole Magnets of the TRISTAN main ring", Proceeding of the 8th Symposium on Accelerator Science and Technology (1991) pp232-233.

[2] Toshiyuki OZAKI, "DCCTs for Magnet Power Supplies", Proceeding of the 10th Symposium on Accelerator Science and Technology (1995) pp106-108.

[3] T. OZAKI, A. AKIYAMA, T. OGITSU, N. OHUCHI, Ta. KUBO, K. TSUCHIYA and M. YOSHIDA, "Power Supplies for Superconducting Magnets at KEKB-IR", Proceeding of the 11th Symposium on Accelerator Science and Technology (1997) pp383385 . 